www.jkns.or.kr

\section{Case Report}

Hyuk Hur, M.D.

Shin Jung, M.D.

Tae-Young Jung, M.D.

In-Young Kim, M.D.

Department of Neurosurgery Chonnam National University Hwasun Hospital \& Medical School, Hwasun, Korea

\title{
Cerebellar Glioblastoma Multiforme in an Adult
}

Primary cerebellar glioblastoma multiforme (GBM) is a rare tumor in adults that accounts for just $1 \%$ of all cases of GBM. Due to their rarity, cerebellar GBMs are not yet completely understood about the pathogenesis and the prognosis. Here, we present a case of GBM in a 69-year-old man. Neurologic examination revealed the presence of cerebellar signs. Magnetic resonance imaging (MRI) showed a 4.5 x $3.6 \mathrm{~cm}$-sized, ill-defined, heterogeneously enhancing mass in the left cerebellum and two patchy hyperintense lesions in the right cerebellum with minimal enhancement. After operation, glioblastoma was histologically confirmed. Postoperative radiotherapy with concomitant and adjuvant temozolomide chemotherapy was subsequently followed. Here, a case of unusual GBM in the cerebellum is reported with review of literature regarding the pathogenesis, the differential diagnosis and prognosis. There was no evidence of recurrence during postoperative one year. This patient showed a good prognosis in spite of the multiple lesions.

KEY WORDS : Cerebellum · Differential diagnosis · Glioblastoma multiforme · Pathogenesis.

\section{INTRODUCTION}

Glioblastoma multiforme (GBM) is the most common primary central nervous (CNS) tumor in adults comprising approximately $50 \%$ of all primary intracranial tumors. They generally occur in the fifth and sixth decades of life. These are infiltrating tumors located in the deep white matter or in the deep gray matter neighboring white matter, primarily in cerebral hemispheres. Primary cerebellar GBM is a rare tumor in adults $\mathrm{s}^{2,6,8,9,15-18)}$ and accounts for just $1 \%$ of all cases of GBM. Cerebellar GBMs are not yet completely understood about the pathogenesis and the prognosis due to their rarity. Here, we report a case of cerebellar GBM in an adult and provide a review of the literature regarding the pathogenesis, diagnosis including radiological and pathological aspects, and treatment of cerebellar GBM.

\section{CASE REPORT}

- Received : May 2, 2007

- Accepted : April 7, 2008

- Address for reprints

Shin Jung, M.D.

Department of Neurosurgery

Chonnam National University

Hwasun Hospital \& Medical School

160 Ilsim-ri, Hwasun-eup

Hwasun-gun, Hwasun 519-809, Korea

Tel : +82-61-379-7666, 7668

Fax : +82-61-379-7673

E-mail : sjung@chonnam.ac.kr
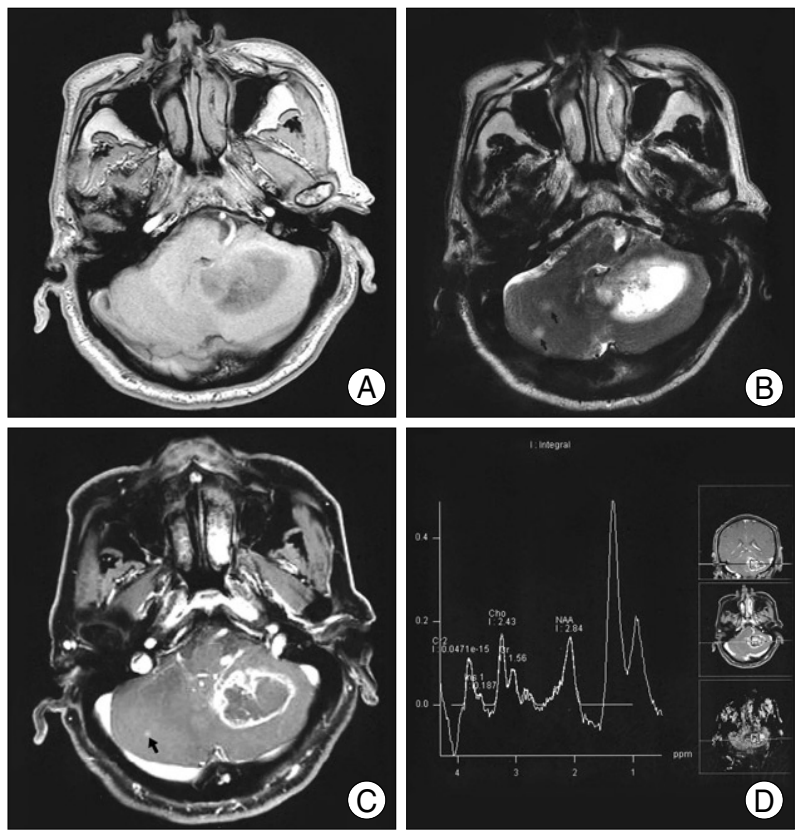

A 69-year-old man presented with headache, dizziness and gait disturbance. Neurological examination revealed the presence of cerebellar signs including cerebellar ataxia, tandem gait, dysmetria and dysdiadochokinesia. He did not have any

Fig. 1. Magnetic resonance (MR) imaging revealed the presence of hypointense and hyperintense lesions in the left cerebellum in T1- and in T2-weighted images, respectively $(A, B)$. The mass had poorly defined borders, mild peritumoral edema, and large areas of central necrosis. T1-weighted MR imaging with Gd-DTPA showed an $4.5 \times 3.6 \mathrm{~cm}$-sized, ill-defined, heterogeneously ring-enhancing with one additional patchy enhancing lesion. (C, Arrow : additional lesion). The MR Spectroscopy showing increased $\mathrm{Cho} / \mathrm{Cr}$ ratio and decreased $\mathrm{N}$-acetil-aspartate peak compatible with a high-grade tumor (D). 

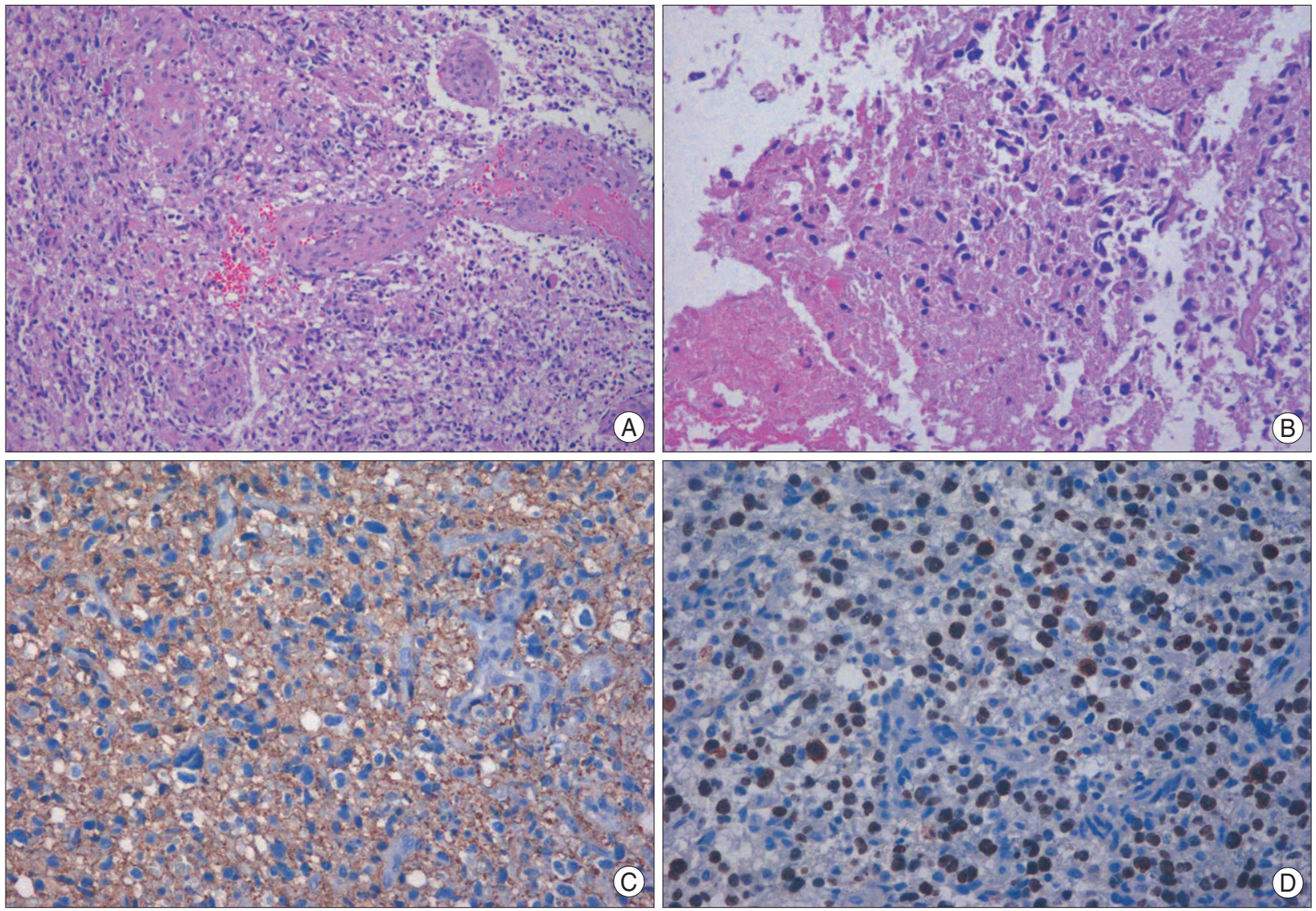

Fig. 2. Histological examination of the removed tumor specimens showed a cellular tumor composed of elongated, spindle-shaped cells with irregular, moderately pleomorphic nuclei, as well as proliferative blood vessels and necrosis (A : original magnification $\times 100$, B : original magnification $\times 200$ ). The tumor cells showed cytoplasmic positivity for glial fibrilary acidic protein (C : original magnification $\times 200)$. Approximately $30-40 \%$ of the nuclei were positive for Ki-67 (D : original magnification $\times 200)$.

past medical history. Magnetic resonance imaging (MRI) showed a hypointense and hyperintense lesion in the left cerebellum on T1- and T2-weighted images, respectively (Fig. 1A, B). The mass had poorly defined borders and mild peritumoral edema. After the gadolinium administration, a $4.5 \times 3.6 \mathrm{~cm}$-sized, ill-defined, heterogeneously ringenhanced mass was observed with one additional patchy enhancing lesion (Fig. 1C). The MR Spectroscopy of the lesion demonstrated a high choline peak (choline/creatinine ratio $=3.3$ ) in contrast to the decreased $\mathrm{N}$-acetil-aspartate (NAA) peak and markedly increased lactate peak, suggesting the presence of a high-grade tumor (Fig. 1D). The provisional diagnosis was metastatic lesion or glioblastoma multiforme (GBM) in the cerebellum. To rule out the metastatic lesion, chest $\mathrm{x}$-ray and abdominal sonography were done but there studies did not show any tumorous lesions.

We performed the operation using the midline suboccipital approach in the prone position. There was a yellowish tumor with necrotic change that had indistinct margins from adjacent normal white matter. A gross total resection was performed. The postoperative course was uneventful and his symptoms and neurologic signs gradually improved. Histological examination showed a cellular tumor, consistent with glioblastoma, composed of elongated, spindle-shaped cells with irregular, moderately pleomorphic nuclei, as well as proliferative blood vessels and necrosis (Fig. 2A, B). The tumor cells showed cytoplasmic positivity for glial fibrillary acidic protein (GFAP) (Fig. 2C). About $30-40 \%$ of the nuclei were positive for Ki-67 (Fig. 3D). The patient received radiotherapy with concomitant and adjuvant temozolomide beginning 24 days after surgery. Irradiation was given 54-Gy dose in total per 27 fractions. There was no evidence of recurrence during postoperative one year.

\section{DISCUSSION}

Glioblastoma (GBM) is the most common primary intracranial tumor in adults. It can be seen in people of all ages. However, approximately two-thirds of the cases occur in adult patients with a peak incidence around the sixth decade ${ }^{16}$ and a second peak in the first decade ${ }^{18)}$. The male to female ratio was $2: 1^{7,8,16}$. 
Infratentorial GBM, however, is a rare entity. Thus, GBM of the cerebellum only comprises a small proportion of all GBM of the brain $(0.24 \text { to } 1.00 \%)^{10)}$. Patients typically present with increased pressure, and the most frequent symptoms are headache, nausea, vomiting and ataxia ${ }^{2,69,15,16,18)}$. Dizziness, neck pain, and mental confusion can also be present $^{9,17,18)}$. The diagnosis of GBM of the cerebellum is not usually suspected preoperatively, although there are certain computed tomography (CT) and MRI features which may point towards it. Cerebellar metastasis and anaplastic astrocytoma are the common differential diagnosis in an adult. Although most authors failed to fully explain the rarity of cerebellar $\mathrm{GBM}^{2,6,16)}$, an explanation for the lesser tendency of cerebellar astrocytes to become malignant has been proposed ${ }^{18)}$.

As stated above, cerebellar astrocytes have lesser tendency to become malignant, but several factors like radiotherapy causing the anaplastic progression of cerebellar astrocytic cells have been proposed. The role of radiotherapy in causing malignant astrocytoma of the cerebellum is uncertain ${ }^{14)}$. But, Maat-Schieman et al. ${ }^{14)}$ reported a midline cerebellar astrocytoma that occurred after radiotherapy following craniopharyngioma. The development of GBM following pilocytic astrocytoma, medulloblastoma and other posterior fossa midline tumors has also been described ${ }^{19,21,22)}$. However, it is difficult to prove their malignant transformation since most of those cases were submitted to radiation therapy postoperatively.

One of the important diagnostic method for cerebellar GBM is CT with contrast enhancement. However, the image of diseases such as metastases, abscess and cerebellar infract is similar with GBM, therefore the differential diagnosis from these diseases is difficult ${ }^{10,15,20)}$. Metastatic tumor is the most common differential diagnosis for posterior fossa brain masses in adults. Solid tumors with contrast enhancement or those with central hypodensity suggesting necrosis were described in cerebellar GBM. Occhiogrosso et al. ${ }^{16)}$ found little peritumoral edema in patients with cerebellar GBM, and Zito et al. ${ }^{23)}$ stated that CT was helpful in differentiating GBM from metastasis of the cerebellum via peritumoral edema or mass effect. The use of MRI has increased the accuracy of the definition of the nature of the lesion because of its good tissue resolution and lack of major bone artifacts ${ }^{2,5}$. The differences in vascularity and metabolite levels in the periphery of a tumor have been found to be significant for the differentiation of metastases and GBMs. The relative cerebral blood volumes in the peritumoral areas calculated with perfusion-weighted MR imaging are clearly higher in gliomas than in metastases. In the spectroscopic MR examination, choline levels are high in the peritumoral areas of gliomas ${ }^{4,12)}$. A significant reduction in NAA due to the loss of neuronal elements, an elevation of the choline peak due to increased membrane synthesis and the presence of a lactate peak all correlate with the higher degree of malignancy commonly observed in GBM.

Abscess shows well-defined, thin-walled enhancing rim on contrast enhanced T1 weighted images. Diffusion weighted image shows increased signal intensity in abscess and ADC map demonstrates markedly decreased signal centrally within abscess. But, primary or metastatic neoplasm is frequently seen as low signal on diffusion weighted images ${ }^{11)}$. The contrast enhancement 1 to 2 weeks after infarction is so called "fogging effect" that is probably due to a reduction in edema and leakage of proteins from cell lysis ${ }^{1}$. However, this lesion shows a virtually normal appearing T2 weighted images.

The biological behavior of cerebellar and supratentorial GBM is similar ${ }^{13,18}$. On the other hand, it is possible that cerebellar GBM found in young aged patients has a better prognosis as in anaplastic astrocytomas (AAs), which have a longer survival time than supratentorial GBM. The median survival for cerebellar GBM is known to be approximately 19 months").

\section{CONCLUSION}

We report an experience of an unusual case of cerebellar GBM. The differential diagnosis with metastases, abscess and cerebellar infract with contrast enhancement is necessary.

\section{References}

1. Asato R, Okumura R, Konishi J : "Fogging effect" in MR of cerebral infarct. J Comput Assist Tomogr 15: 160-162, 1991

2. Aun RA, Stavale JN, Silva-Junior D : [Glioblastoma multiforme of the cerebellum. Report of a case]. Arq Neuropsiquiatr $39: 350-354$, 1981

3. Bilaniuk LT : Adult infratentorial tumors. Semin Roentgenol 25 : 155173,1990

4. Demir MK, Hakan T, Akinci O, Berkman Z : Primary cerebellar glioblastoma multiforme. Diagn Interv Radiol 11 : 83-86, 2005

5. Gusnard DA : Cerebellar neoplasm in children. Semin Roentgenol 25 : 263-278, 1990

6. Hegedüs K, Molnár P : Primary cerebellar glioblastoma multiforme with an unusually long survival. Case report. J Neurosurg 58 : 589592, 1983

7. Joung YI, Cheong JH, Bak KH : Cerebellar Glioblastoma Presenting as a Cerebellar Hemorrhage in a Child, J Korean Neurosurg Soc 39 : 374-377, 2006

8. Kopelson G : Cerebellar glioblastoma. Cancer 50 : 308-311, 1982

9. Kulkarni AV, Becker LE, Jay V, Armstrong DC, Drake JM : Primary cerebellar glioblastomas multiforme in children. Report of four cases. J Neurosurg $90: 546-550,1999$

10. Kuroiwa T, Numaguchi Y, Rothman MI, Zoarski GH, Morikawa M, Zagardo MT, et al : Posterior fossa glioblastoma multiforme : MR findings. AJNR Am J Neuroradiol 16 : 583-589, 1995

11. Lai PH, Ho JT, Chen WL, Hsu SS, Wang JS, Pan HB, et al : Brain abscess and necrotic brain tumor : discrimination with proton MR spectroscopy and diffusion-weighted imaging. AJNR Am J Neuroradiol $23: 1369-1377,2002$

12. Law M, Cha S, Knopp EA, Johnson G, Arnett J, Litt AW : High-grade 
gliomas and solitary metastases : differentiation by using perfusion and proton spectroscopic MR imaging. Radiology 222 : 715-721, 2002

13. Luccarelli $\mathrm{G}$ : Glioblastoma multiforme of the cerebellum : description of three cases. Acta Neurochir (Wien) $53: 107-116,1980$

14. Maat-Schieman ML, Bots GT, Thomeer RT, Vielvoye GJ : Malignant astrocytoma following radiotherapy for craniopharyngioma. $\mathrm{Br} \mathbf{J}$ Radiol 58 : 480-482, 1985

15. Miller EM, Mani RL, Townsend JJ : Cerebellar glioblastoma multiforme in an adult. Surg Neurol $5: 341-343,1976$

16. Occhiogrosso M, Spada A, Merlicco G, Vailati G, De Benedictis G : Malignant cerebellar astrocytoma. Report of five cases. J Neurosurg Sci $29: 43-50,1985$

17. Pombo R, Tortelly-Costa A, Bulacio E, Hahn MD, de Carvalho ML : [Cerebellar glioblastoma multiforme : report of a case in a child]. Arq Neuropsiquiatr $43: 102-107,1985$

18. Rosenfeld J, Rossi ML, Briggs M : Glioblastoma multiforme of the cerebellum in an elderly man. A case report. Tumori 75 : 626-629, 1989

19. Schmidbauer M, Budka H, Bruckner R, Vorkapic P : Glioblastoma developing at the site of a cerebellar medulloblastoma treated 6 years earlier. Case report. J Neurosurg 67 : 915-918, 1987

20. Tamaki T, Eguchi T, Sakamoto M, Teramoto A : Glioblastoma multiforme of the cerebellum in an elderly man. J Chin Med Assoc 67:301-304, 2004

21. Ushio Y, Arita N, Yoshimine T, Ikeda T, Mogami H : Malignant recurrence of childhood cerebellar astrocytoma : case report. Neurosurgery $21: 251-255,1987$

22. Wisoff HS, Llena JF : Glioblastoma multiforme of the cerebellum five decades after irradiation of a cerebellar tumor. J Neurooncol 7 : 339-344, 1989

23. Zito JL, Siva A, Smith TW, Leeds M, Davidson R : Glioblastoma of the cerebellum. Computed tomographic and pathologic considerations. Surg Neurol $19: 373-378,1983$ 